This item was submitted to Loughborough's Research Repository by the author.

Items in Figshare are protected by copyright, with all rights reserved, unless otherwise indicated.

\title{
Scattering and collision of gravitational waves in Friedmann-Robertson- Walker open universes
}

PLEASE CITE THE PUBLISHED VERSION

PUBLISHER

(C) American Physical Society

LICENCE

CC BY-NC-ND 4.0

REPOSITORY RECORD

Bicak, Jiri, and J.B. Griffiths. 2019. "Scattering and Collision of Gravitational Waves in Friedmann-robertsonwalker Open Universes”. figshare. https://hdl.handle.net/2134/1713. 


\title{
Scattering and collision of gravitational waves in Friedmann-Robertson-Walker open universes
}

\author{
Jiří Bičák \\ Department of Theoretical Physics, Faculty of Mathematics and Physics, Charles University, \\ $V$ Holešovičkách 2, 18000 Prague 8, Czech Republic \\ Jerry B. Griffiths \\ Department of Mathematical Sciences, University of Technology, Loughborough, Leicestershire LE11 3TU, United Kingdom
}

(Received 16 August 1993)

\begin{abstract}
An exact solution is presented which describes the scattering of a gravitational wave in an open expanding universe. The background region ahead of the wave is taken to be a Friedmann-RobertsonWalker $(k=-1)$ space-time containing a perfect fluid with an extreme relativistic equation of state $p=\rho$. Plane surfaces are determined in this background, so that solutions describing the scattering of a single wave with a wave front given by such a surface and the collision of two such waves can be obtained.
\end{abstract}

PACS number(s): $04.40 . \mathrm{Nr}, 04.30 . \mathrm{Nk}$

\section{INTRODUCTION}

Most work on gravitational radiation in terms of exact solutions of Einstein's equations has been concentrated on space-times which are either flat asymptotically or contain flat regions explicitly. Boost-rotation symmetric solutions represent examples of exact radiative spacetimes which are "almost everywhere" asymptotically flat (see [1] for a review of exact radiative space-times). The sandwich plane waves and the great majority of solutions describing colliding plane waves possess flat Minkowski regions (for a comprehensive review see [2]). Considerably less attention has been paid to the propagation and interaction of gravitational waves in cosmological models.

The collision of plane gravitational waves in a vacuum Kasner background has been analyzed by Centrella and Matzner $[3,4]$ by combining analytical estimates with numerical computation. Exact solutions describing socalled gravitational "solitons" have also been investigated in a cosmological context (see [5] and references contained therein, and also [6]). However, these solutions have dealt only with the vacuum case. Similar solutions containing scalar and electromagnetic waves have also been obtained (for a review see [7]), but little progress has been made on the combination of gravitational waves with perfect fluids. One exact formalism [8] was developed for gravitational waves in backgrounds corresponding to Bianchi types I-VII. However, the only explicit solution given is again a vacuum case.

For perfect fluid backgrounds, the simplest case to consider is that of the so-called "stiff" perfect fluid in which the equation of state is at the extreme relativistic limit $p=\rho$. This constraint implies that the speed of sound is the same as that of light, so the characteristics of the governing equations are the same as those of the gravitational field. In this case the characteristic surfaces are null and it is possible to consider gravitational waves propagating along them. In this situation, classes of ex- act solutions are attainable.

The propagation of fluid shocks in such a fluid has been considered by Tabensky and Taub [9]. However, our purpose here is to consider the propagation of free gravitational waves. We will, therefore, attempt to exclude fluid shock waves.

An interesting exact solution describing the propagation of a gravitational wave pulse through a fluid in which $p=\rho$ has previously been given by Wainwright [10]. In this case, the gravitational field ahead of and behind the wave is of type $D$, and the gravitational wave has variable polarization in which the polarization is not independent of the amplitude.

In order to further explore the passage of a purely gravitational wave in a fluid background, one of us has recently considered the problem of the scattering of a gravitational wave as it propagates through a perfect fluid with $p=\rho[11]$, and also the collision of waves in such a medium [12]. In that case the background region into which the waves propagate was taken to be a Friedmann-Robertson-Walker (FRW) $k=0$ space-time. The purpose of the present paper is to show how the previous work can be generalized to include alternative, more complicated backgrounds, and to discuss the collision of waves in an expanding universe in greater detail.

In the previous work, with the background being the FRW $k=0$ space-time, the spatial sections are flat and null planes can easily be defined. The first problem considered here is how to describe appropriate plane wave surfaces in the other FRW universes in which the spatial sections are curved. It will be argued that such surfaces cannot be obtained in the closed universes with $k=1$, but that they can in the open FRW universes with $k=-1$. The FRW universes are particularly appropriate backgrounds to consider since they are conformally flat. This permits the features of free gravitational waves, as represented by the components of the Weyl tensor, to be clearly identified. 
For simplicity, the gravitational waves that are considered to propagate into such a background are taken to have constant linear polarization. It will be shown that such waves are necessarily backscattered, so that the region behind the waves is algebraically general. The collision and subsequent interaction of such waves will also be considered. It will be indicated, by analyzing the expansion of the fluid, that in expanding open universes colliding gravitational waves do not apparently produce singularities.

\section{PLANES AND NULL HYPERSURFACES IN THE FRIEDMANN-ROBERTSON-WALKER OPEN UNIVERSES}

The standard form of the metric of an open FRW model with negative spatial curvature can be written in coordinates $\chi \in[0,+\infty), \theta \in[0, \pi), \phi \in[0,2 \pi)$ as

$d s^{2}=d t^{2}-R^{2}(t)\left[d \chi^{2}+\sinh ^{2} \chi\left(d \theta^{2}+\sin ^{2} \theta d \phi^{2}\right)\right]$.

The spatial three-geometry on a hypersurface $t=t_{0}=$ constant is, up to a constant factor $R^{2}\left(t_{0}\right)$, given by

$$
d \ell^{2}=d \chi^{2}+\sinh ^{2} \chi\left(d \theta^{2}+\sin ^{2} \theta d \phi^{2}\right) .
$$

It cannot be embedded in a four-dimensional Euclidean space but it can be embedded in a four-dimensional Minkowski space (see, e.g., [13]),

$$
d \sigma^{2}=d V^{2}-d X^{2}-d Y^{2}-d Z^{2}
$$

the embedded surface being a three-dimensional hyperboloid:

$$
V^{2}-X^{2}-Y^{2}-Z^{2}=1
$$

By setting

$$
\begin{aligned}
& X=\sinh \chi \sin \theta \cos \phi, \\
& Y=\sinh \chi \sin \theta \sin \phi, \\
& Z=\sinh \chi \cos \theta, \\
& V=\cosh \chi
\end{aligned}
$$

it is straightforward to see that (2.4) is satisfied, and (2.3) $-(2.5)$ imply $d \sigma^{2}=-d \ell^{2}$, where $d \ell^{2}$ is given by (2.2).

We can introduce another system of spatial coordinates on the three-hyperboloid (2.4) such that the three-metric is conformally flat. By putting

$$
\begin{aligned}
& X=\frac{x}{z}, \quad Y=\frac{y}{z} \\
& Z=\frac{1}{2 z}\left(1-x^{2}-y^{2}-z^{2}\right) \\
& V=\frac{1}{2 z}\left(1+x^{2}+y^{2}+z^{2}\right)
\end{aligned}
$$

where $x, y \in(-\infty, \infty), z \in(0,+\infty)$, we find (2.4) to be satisfied. Restricting ourselves to $z>0$, our new coordinate system $(x, y, z)$ sweeps out the whole "positive" $(V>0)$ sheet of the hyperboloid (2.4). Inserting (2.6) into (2.3), we obtain the metric on the hyperboloid in the conformally flat form

$$
-d \sigma^{2}=d \ell^{2}=\frac{1}{z^{2}}\left(d x^{2}+d y^{2}+d z^{2}\right) .
$$

Now consider the sections of the hyperboloid (2.4) given by $z=z_{0}=$ const. Equations (2.6) then imply $X=x / z_{0}, Y=y / z_{0}$, so that $(X, Y) \in \mathbb{R}^{2},(x, y) \in \mathbb{R}^{2}$, and $V+Z=1 / z_{0}$. Hence, we are just cutting the threehyperboloid (2.4) by "null hyperplanes" $V+Z=$ const in Minkowski space (2.3). Since then $d V=-d Z,(2.3)$ immediately implies that the two-dimensional sections we obtain are spacelike and their intrinsic geometry is flat. The same result, of course, follows directly from (2.7) by putting $z=$ const therein. In choosing coordinates according to (2.6), we "singled out" the coordinate $z$ (while $x, y$ are appearing symmetrically), and thus also the set of two-planes given by $z=$ const. However, since the threehyperboloid (2.4) is isotropic and homogeneous, we can construct other sets of two-planes by simple transformations.

Notice that the geometry of the two-spaces $z=$ const would not be flat if the three-hyperboloid (2.4) would be embedded in a four-dimensional Euclidean space rather than in Minkowski space. In the case of the FRW models with positive spatial curvature the hypersurfaces $t=$ constant are three-dimensional spheres in four-dimensional Euclidean space (cf., e.g., [13]). No analogous set of planes can be constructed in this case.

Returning now to the space-time metric (2.1), we go over from coordinates $(\chi, \theta, \phi)$ to new coordinates $(x, y, z)$ by the transformation given by combining $(2.5)$ and (2.6):

$$
\begin{aligned}
\sinh \chi \sin \theta \cos \phi & =\frac{x}{z}, \\
\sinh \chi \sin \theta \sin \phi & =\frac{y}{z} \\
\sinh \chi \cos \theta & =\frac{1}{2 z}\left(1-x^{2}-y^{2}-z^{2}\right),
\end{aligned}
$$

which implies that $\cosh \chi=(1 / 2 z)\left(1+x^{2}+y^{2}+z^{2}\right)$. It is easy to express $(\chi, \theta, \phi)$ directly in terms of $(x, y, z)$ and vice versa. Under this transformation the FRW line element (2.1) becomes

$$
d s^{2}=d t^{2}-R^{2}(t)\left[\frac{1}{z^{2}}\left(d x^{2}+d y^{2}+d z^{2}\right)\right] .
$$

Introducing next the new coordinate

$$
\mu=\ln z
$$

we obtain the metric

$$
d s^{2}=d t^{2}-R^{2}(t)\left[d \mu^{2}+e^{-2 \mu}\left(d x^{2}+d y^{2}\right)\right] .
$$

Finally, going over to the "conformal-time" coordinate $\eta$ by the usual relation

$$
d t=R(\eta) d \eta
$$

we arrive at the metric

$$
d s^{2}=R^{2}(\eta)\left[d \eta^{2}-d \mu^{2}-e^{-2 \mu}\left(d x^{2}+d y^{2}\right)\right] .
$$


It is now easy to find two families of null hypersurfaces by putting

$$
\eta=\tilde{u}+\tilde{v}, \quad \mu=\tilde{u}-\tilde{v}
$$

or

$$
\tilde{u}=\frac{1}{2}(\eta+\mu), \quad \tilde{v}=\frac{1}{2}(\eta-\mu) .
$$

The metric then reads

$$
d s^{2}=R^{2}(\tilde{u}+\tilde{v})\left[4 d \tilde{u} d \tilde{v}-e^{-2(\tilde{u}-\tilde{v})}\left(d x^{2}+d y^{2}\right)\right],
$$

with $\tilde{u}=$ const and $\tilde{v}=$ const being the two families of null hypersurfaces. These intersect in two-planes with $(x, y) \in \mathbb{R}^{2}$.

\section{FRIEDMANN-ROBERTSON-WALKER MODELS WITH A FLUID WITH A STIFF EQUATION OF STATE}

It is simple to integrate Einstein's field equations for the FRW models filled with a perfect fluid satisfying the extremely relativistic equation of state $p=\rho$. Although various spatially homogeneous and even inhomogeneous cosmologies with such a "stiff" fluid have been analyzed in the literature [14], we have not found the simplest FRW cases given explicitly. For the benefit of the reader, these are now stated. We treat all three possibilities of the FRW universes with the positive $(k=+1)$, zero $(k=$ $0)$, and negative $(k=-1)$ spatial curvature.

With $p=\rho$, the equation of energy conservation, $(\rho R)_{, t}=-p\left(R^{3}\right)_{, t}$, implies that

$$
\rho R^{6}=\rho_{0} R_{0}^{6}=\text { const }=\gamma_{0} .
$$

Substituting for $\rho$ into the only field equation which remains to be satisfied (e.g., $[13]),\left(R_{, t} / R\right)^{2}=-k / R^{2}+$ $(8 \pi / 3) \rho$, we obtain

$$
R_{, t}^{2}=-k+\gamma^{2} R^{-4}
$$

where $\gamma^{2}=(8 \pi / 3) \gamma_{0}=$ const. [Notice that, in the geometrical units we are using here, the dimension of $\gamma$ is (length) ${ }^{2}, R$ and $t$ have the dimension of length, while $\eta, \mu, x$, and $y$ are dimensionless.] Introducing the conformal-time coordinate $\eta$ in accordance with (2.12), Eq. (3.2) converts into

$$
R_{, \eta}=\left[-k R^{2}+\gamma^{2} R^{-2}\right]^{\frac{1}{2}}
$$

This can easily be integrated to yield

$$
\begin{array}{ll}
R=[\gamma \sinh 2 \eta]^{\frac{1}{2}} & \text { if } k=-1, \\
R=[\gamma 2 \eta]^{\frac{1}{2}} & \text { if } k=0, \\
R=[\gamma \sin 2 \eta]^{\frac{1}{2}} & \text { if } k=+1 .
\end{array}
$$

Here we have put an integration constant equal to zero, so that the big-bang singularity occurs at $\eta=0$.

In order to obtain the expansion function $R$ as a func- tion of the "cosmic time" $t$, we have to find $t=t(\eta)$ by integrating Eq. (2.12) with $R$ given in (3.4), and express back $\eta=\eta(t)$. In the simplest case of $k=0$ we immediately get

$$
R=(3 \gamma t)^{\frac{1}{3}} .
$$

Hence, the flat FRW universe with a "stiff" fluid expands slower than the corresponding universe with dust for which $R \sim t^{\frac{2}{3}}$ (e.g., [13]).

In the more complicated cases with $k= \pm 1$, we can still explicitly integrate Eq. (2.12) to find (see [15], expression 2.464.5) for $k=-1$ :

$$
\begin{aligned}
t=\sqrt{\gamma}\left[\frac{1}{2} F\right. & \left(\alpha, \frac{1}{\sqrt{2}}\right)-E\left(\alpha, \frac{1}{\sqrt{2}}\right) \\
& \left.+\frac{\left[\sinh 2 \eta\left(1+\sinh ^{2} 2 \eta\right)\right]^{\frac{1}{2}}}{1+\sinh 2 \eta}\right],
\end{aligned}
$$

where $\alpha=\arccos [(1-\sinh \eta) /(1+\sinh \eta)]$, and $F$ and $E$ are the elliptic integrals of the first and the second kind, respectively. If $k=+1$, we obtain (see [15], 2.619.11 with $x \rightarrow x^{\prime}-\pi / 4$ )

$$
t=\sqrt{\frac{\gamma}{2}}\left[F\left(\alpha, \frac{1}{\sqrt{2}}\right)-2 E\left(\alpha, \frac{1}{\sqrt{2}}\right)\right]_{\frac{\pi}{4}}^{\frac{\pi}{4}+\eta},
$$

where $\alpha=\arccos [\sqrt{2} \cos (\eta+\pi / 4)]$, and $F$ and $E$ are again elliptic integrals. Therefore, in these cases, $R(t)$ can be found only in parametric forms given by (3.4), (3.6), and (3.7).

Notice that for the negative curvature open universe, in which we are primarily interested here, Eq. (3.7) implies that $t \approx(\gamma \sinh 2 \eta)^{\frac{1}{2}}$ as $\eta \rightarrow \infty$. Hence, at large times the $k=-1$ FRW model with a stiff fluid expands linearly,

$$
R \cong t
$$

i.e., exactly as the standard $k=-1$ Friedmann models with dust or radiation. This, of course, was to be expected, since the Einstein equation from which we started implies that, asymptotically, at large $R$ 's the universe with $k=-1$ is just "curvature dominated."

\section{THE FIELD EQUATIONS IN NULL COORDINATES}

The purpose of this section is to present field equations for the scattering or interaction of gravitational waves in a fluid with a stiff equation of state in an appropriate coordinate system. In order to find solutions that can be matched to the background space-times considered above across a null hypersurface, it is assumed that there exist two Killing vectors $\partial_{x}$ and $\partial_{y}$. Restricting attention to the case in which any gravitational wave has a fixed polarization and colliding waves have collinear polarization, the line element can be taken in the form

$$
d s^{2}=2 e^{-M} d u d v-e^{-U}\left(e^{V} d x^{2}+e^{-V} d y^{2}\right),
$$

where $U, V$, and $M$ are functions of the null coordinates 
$u$ and $v$ only. A standard null tetrad $(l, n, m, \bar{m})$ can always be chosen such that the two real null vectors are

$$
l_{\mu}=e^{-M / 2} u,_{\mu}, \quad n_{\mu}=e^{-M / 2} v_{\mu},
$$

and the null vectors $m_{\mu}$ and $\bar{m}_{\mu}$ are combinations of the two Killing vectors. In the solution described below, it is assumed that the fluid does not move in the directions spanned by $\partial_{x}$ and $\partial_{y}$, and it is thus possible to express the fluid four-velocity as

$$
u^{\mu}=\frac{1}{\sqrt{2}}\left(a l^{\mu}+b n^{\mu}\right)
$$

where $a$ and $b$ are functions of $u$ and $v$ only, and $a b=1$.

It is well known that a stiff perfect fluid for which the pressure $p$ is equal to the density $\rho$ can be related to a scalar or potential field [16] which is here described by a function $\sigma$. Using the metric defined by (4.1), $\sigma(u, v)$ is required to satisfy the equation (representing the contracted Bianchi identities)

$$
2 \sigma_{u v}=U_{u} \sigma_{v}+U_{v} \sigma_{u} .
$$

In terms of this function, the fluid density and fourvelocity components are given by

$$
\begin{aligned}
16 \pi \rho & =e^{M} \sigma_{u} \sigma_{v}, \\
a=\sqrt{\sigma_{u} / \sigma_{v}}, & b=\sqrt{\sigma_{v} / \sigma_{u}},
\end{aligned}
$$

which ensures that the fluid four-velocity is proportional to the gradient of $\sigma$. In addition, the nonzero components of the Ricci tensor are given in Newman-Penrose notation by

$$
\begin{gathered}
\Phi_{00}=\frac{1}{4} e^{M}{\sigma_{v}}^{2}, \quad \Phi_{11}=-3 \Lambda=\frac{1}{8} e^{M} \sigma_{u} \sigma_{v} \\
\Phi_{22}=\frac{1}{4} e^{M}{\sigma_{u}}^{2} .
\end{gathered}
$$

The field equations can now be written in the suitable form

$$
\begin{aligned}
U_{u v} & =U_{u} U_{v} \\
2 U_{v v} & =U_{v}^{2}+V_{v}^{2}-2 U_{v} M_{v}+{\sigma_{v}}^{2}, \\
2 U_{u u} & =U_{u}^{2}+V_{u}^{2}-2 U_{u} M_{u}+{\sigma_{u}}^{2}, \\
2 M_{u v} & =-U_{u} U_{v}+V_{u} V_{v}+\sigma_{u} \sigma_{v} \\
2 V_{u v} & =U_{u} V_{v}+U_{v} V_{u} .
\end{aligned}
$$

The nonzero components of the Weyl tensor, which represent the gravitational waves (free gravitational field components), can be expressed as

$$
\begin{aligned}
& \Psi_{0}=-\frac{1}{2} e^{M}\left(V_{v v}-U_{v} V_{v}+M_{v} V_{v}\right) \\
& \Psi_{2}=\frac{1}{2} e^{M} M_{u v}-\frac{1}{6} e^{M} \sigma_{u} \sigma_{v}, \\
& \Psi_{4}=-\frac{1}{2} e^{M}\left(V_{u u}-U_{u} V_{u}+M_{u} V_{u}\right) .
\end{aligned}
$$

It can be seen that Eq. (4.7) can immediately be integrated to give

$$
e^{-U}=f(u)+g(v)
$$

where $f(u)$ and $g(v)$ are arbitrary functions. It is conve- nient here to choose the gauge such that

$$
e^{-U}=u+v
$$

It should also be observed that Eqs. (4.4), (4.7), and (4.11) are the integrability conditions for Eqs. (4.8), (4.9), and (4.10). Thus if Eqs. (4.4) and (4.11) are satisfied, there automatically exists a function $M$ which satisfies the remaining equations, and which can be obtained by a simple quadrature.

Let us now express the FRW space-time with $k=-1$, and which contains a stiff fluid, in this notation. Substituting from Eq. (3.4) into the metric (2.16) yields the line element

$$
\begin{aligned}
d s^{2}=2 \gamma & {\left[e^{2(\tilde{u}+\tilde{v})}-e^{-2(\tilde{u}+\tilde{v})}\right] d \tilde{u} d \tilde{v} } \\
& -\frac{1}{2} \gamma\left(e^{4 \tilde{v}}-e^{-4 \tilde{u}}\right)\left(d x^{2}+d y^{2}\right)
\end{aligned}
$$

Rescaling the coordinates $x$ and $y$ such that $x \rightarrow x^{\prime}=$ $\sqrt{\gamma / 2} x$ and $y \rightarrow y^{\prime}=\sqrt{\gamma / 2} y$ (but omitting the primes), and introducing new null coordinates by the relations

$$
u-c=-e^{-4 \tilde{u}}, \quad v+c=e^{4 \tilde{v}}
$$

where $c>0$ is an arbitrary constant, the metric (4.15) is recast in the form

$$
d s^{2}=\frac{\gamma}{16} \frac{u+v}{(c-u)^{\frac{3}{2}}(c+v)^{\frac{3}{2}}} 2 d u d v-(u+v)\left(d x^{2}+d y^{2}\right) .
$$

Hence, in terms of the functions $U, M$, and $V$ introduced above, we obtain

$$
\begin{aligned}
U & =-\ln (u+v), \\
M & =-\ln (u+v)+\frac{3}{2} \ln (c-u)+\frac{3}{2} \ln (c+v)-\ln \left(\frac{\gamma}{16}\right), \\
V & =0 .
\end{aligned}
$$

Part of this space-time is represented in terms of these coordinates in Fig. 1.

It is easy to see that the potential

$$
\sigma=\sqrt{3} \ln \left(\frac{\sqrt{c+v}-\sqrt{c-u}}{\sqrt{c+v}+\sqrt{c-u}}\right)
$$

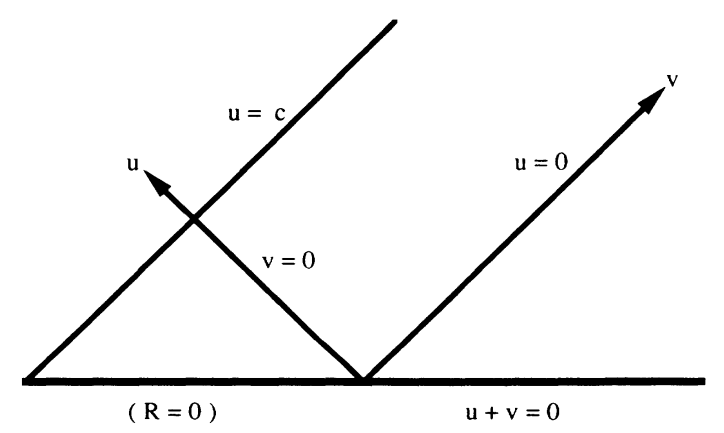

FIG. 1. The coordinate range for the FRW open $(k=-1)$ universe with a stiff fluid as represented by the metric (4.17). The spacelike surface $u+v=0$ corresponds to the initial big-bang singularity, and $u=c$ corresponds to part of null infinity. 
satisfies Eqs. (4.4) and (4.8)-(4.10). From this, and from (4.5), we obtain

$$
\rho=3 \frac{(c-u)^{3 / 2}(c+v)^{3 / 2}}{\pi \gamma(u+v)^{3}},
$$

which clearly indicates the "big-bang" curvature singularity at $u+v=0$ and the timelike infinity at $u \rightarrow c$, $v \rightarrow \infty$ [cf. (2.15) and (4.16)]. Finally, we note that the four-velocity of the fluid is given by (4.3) in which

$$
a=\left(\frac{c+v}{c-u}\right)^{1 / 2}, \quad b=\left(\frac{c-u}{c+v}\right)^{1 / 2} .
$$

In the form of the metric given by (4.17), it is evident that the surfaces given by $u=$ const and $v=$ const are plane. It is therefore possible to consider hypersurfaces $u=\operatorname{const}(<c)$, or alternatively $v=$ const, as wave fronts in which the above solution is chosen ahead of the wave. The region containing the wave will then have to be matched to this solution across such a hypersurface.

To consider such a situation, it is convenient to initially assume that the gravitational wave can be described by the line element (4.1). To match this to a background region described by the metric (4.17) across a null hypersurface given by $u=$ const or $v=$ const, it is necessary that $U$ is smooth across the hypersurface and that $V, M$, and $\sigma$ are continuous [17].

\section{SCATTERING OF A PLANE GRAVITATIONAL WAVE IN A FRW OPEN UNIVERSE}

Consider now the propagation of a gravitational wave with wave front given by the null hypersurface $u=0$. The background region of space-time ahead of the wave is part of the conformally flat FRW $(k=-1)$ universe described above with $u<0$. This will be referred to as region $\mathrm{I}$.

The region containing the wave, for which $u \geq 0$, will be referred to as region II (see Fig. 2). It will be assumed

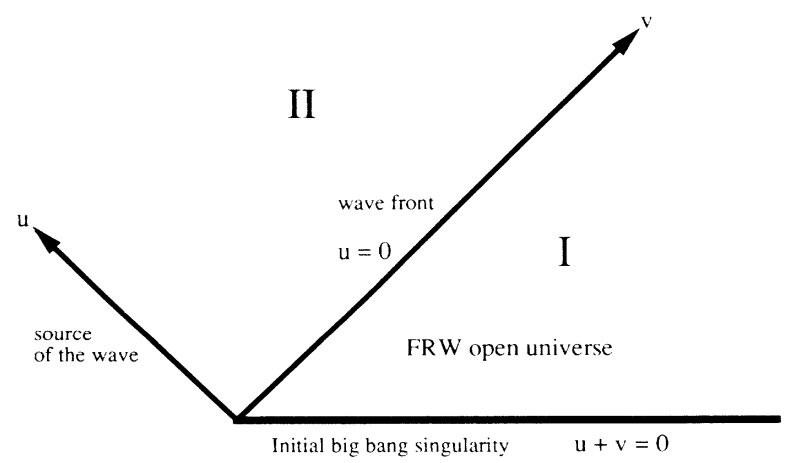

FIG. 2. Region I is part of the open FRW universe. It contains a perfect fluid expanding from an initial singularity at $u+v=0$. Into this a gravitational wave propagates with wave front $u=0$. This wave is partially backscattered and region II is algebraically general. Our solution breaks down at $v=0$ in region II, which is where the sources of the wave are to be expected. that the line element in this region can be taken in the form (4.1). This implies that the gravitational wave has constant linear polarization.

We can continue to adopt a gauge in region II such that

$$
e^{-U}=u+v
$$

which is the same as that in region I. This ensures that $U$ is smooth across the wave fronts. We can also take $\sigma$ to have the same functional form in both regions $\mathrm{I}$ and II: namely [cf. Eq. (4.19)],

$$
\sigma=\sqrt{3} \ln \left(\frac{\sqrt{c+v}-\sqrt{c-u}}{\sqrt{c+v}+\sqrt{c-u}}\right) .
$$

This ensures that the fluid remains continuous across the wave fronts, thus automatically avoiding the introduction of fluid shocks. With these initial assumptions, part of future null infinity is again given by $u \rightarrow c$, so that $u$ is confined to the range $0 \leq u<c$.

It is now necessary to obtain a solution for $V(u, v)$ satisfying Eq. (4.11). With (5.1), this can be written as a Euler-Darboux equation with noninteger coefficients:

$$
(u+v) V_{u v}+\frac{1}{2} V_{u}+\frac{1}{2} V_{v}=0 .
$$

A solution of this equation is required such that $V=0$ and $V_{u}$ is bounded on the wave front $u=0$. Such a solution has been discussed elsewhere [11] and may be written in the form

$$
\begin{aligned}
V & =c_{n} \frac{u^{n}}{\sqrt{v}} F\left(\frac{1}{2}, \frac{1}{2}+n ; 1+n ;-\frac{u}{v}\right) \\
& =c_{n} \frac{u^{n}}{\sqrt{u+v}} F\left(\frac{1}{2}, \frac{1}{2} ; 1+n ; \frac{u}{u+v}\right),
\end{aligned}
$$

where $n \geq 1$ and $c_{n}$ are arbitrary constants, and $F\left(\frac{1}{2}, \frac{1}{2}+n ; 1+n ;-u / v\right)$ and $F\left(\frac{1}{2}, \frac{1}{2} ; 1+n ; \frac{u}{u+v}\right)$ are hypergeometric functions. It may be noticed that near the wave front, $V$ develops as $u^{n}$.

Since Eq. (5.3) is linear, any solutions of the form (5.4) with different values of $n$ and $c_{n}$ may be combined to obtain arbitrary profiles of the gravitational wave.

The remaining metric function can be determined by first putting

$M_{0}=-\ln (u+v)+\frac{3}{2} \ln (c-u)+\frac{3}{2} \ln (c+v)-\ln \left(\frac{\gamma}{16}\right)$,

which is the expression for $M$ in region I [see Eq. (4.18)]. It is now possible to obtain $M$ in region II by putting

$$
M=M_{0}+\Omega,
$$

where $\Omega(u, v)$ is zero on $u=0$ and satisfies the equations [see (4.8) and (4.9)]

$$
\Omega_{v}=-\frac{1}{2}(u+v) V_{v}^{2}, \quad \Omega_{u}=-\frac{1}{2}(u+v) V_{u}^{2} .
$$

We have thus determined the solution up to this quadrature. by 


$$
\begin{aligned}
& \Psi_{0}=-\frac{1}{4} e^{M}\left(2 V_{v v}+\frac{3}{(c+v)} V_{v}-(u+v) V_{v}^{3}\right) \\
& \Psi_{2}=\frac{1}{4} e^{M} V_{u} V_{v} \\
& \Psi_{4}=-\frac{1}{4} e^{M}\left(2 V_{u u}-\frac{3}{(c-u)} V_{u}-(u+v) V_{u}^{3}\right) .
\end{aligned}
$$

It should immediately be noticed that the propagating wave which is represented essentially by the component $\Psi_{4}$ is partially reflected, so that the gravitational field behind the wave front is algebraically general. It can be easily seen that, when $n=1$ or $n=2$ in (5.4), the component $\Psi_{4}$ has a step wave front. For higher values of $n, \Psi_{4}$ develops as $u^{n-2}$ while $\Psi_{0}$ which describes the backscattered wave develops as $u^{n}$. The $\Psi_{2}$ component develops as $u^{2 n-1}$.

It should also be noticed that the hypergeometric functions appearing in (5.4) are not defined for $(-u / v) \rightarrow \infty$ (cf., e.g., [18]). Thus, this region behind the wave may contain singularities which could be considered as the sources of the gravitational wave. The form of the sources will be determined by the set of constants $c_{n}$.

\section{COLLISIONS OF PLANE WAVES IN A FRIEDMANN-ROBERTSON-WALKER BACKGROUND}

In recent years considerable attention has been paid to the problem of the collision and interaction of plane gravitational waves. Numerous classes of exact solutions have been obtained and their singularity structure has been clarified. A substantial review of this work has recently been given in [2]. However, in all of this work the background region ahead of the waves prior to the collision has been taken to be flat Minkowski space.

Part of the motivation for the present work has been to consider the colliding wave problem in the context of a cosmological background. With the results of the previous sections, we can now consider the collision of gravitational waves in a FRW $(k=-1)$ background containing

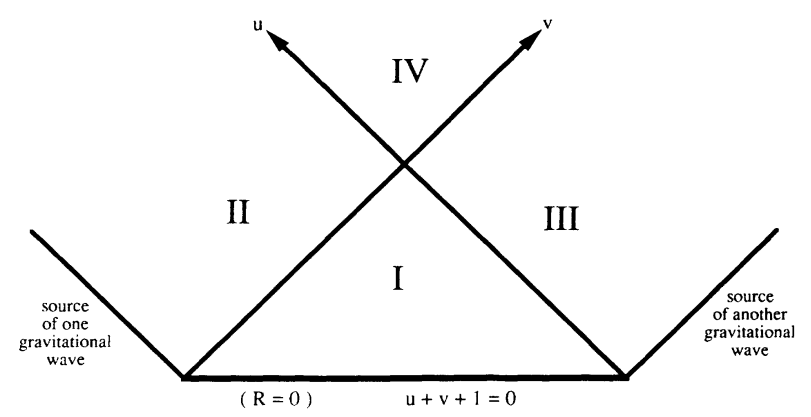

FIG. 3. The background region $I$ is part of the FRW $(k=-1)$ space-time. It contains a stiff perfect fluid expanding from an initial singularity. Into this background, gravitational waves in regions II and III propagate with wave fronts $u=0$ and $v=0$. Region IV is the interaction region following the collision. Regions II, III, and IV are all algebraically general. a perfect fluid with equation of state $p=\rho$.

With the formulation of the FRW $(k=-1)$ space-time given above, we can consider approaching plane gravitational waves propagating along the hypersurfaces $u=$ const and $v=$ const. At this point it is convenient to replace $v$ by $1+v$ in the formulas of previous sections so that we can consider approaching waves with wave fronts given by $u=0$ and $v=0$. The various regions of the space-time are illustrated in Fig. 3. The initial singularity $(R=0)$ now occurs on the hypersurface $u+v+1=0$.

The background region $I$ is FRW space-time with $k=-1$. It is described by modifying the solution (4.18)(4.21) to the form

$$
\begin{aligned}
& U=U_{0}=-\ln (1+u+v), \\
& V=0 \text {, } \\
& M=M_{0}=-\ln (1+u+v)+\frac{3}{2} \ln (c-u) \\
& +\frac{3}{2} \ln (c+1+v)-\ln \left(\frac{\gamma}{16}\right) \text {, } \\
& \sigma=\sigma_{0}=\sqrt{3} \ln \left(\frac{\sqrt{c+1+v}-\sqrt{c-u}}{\sqrt{c+1+v}+\sqrt{c-u}}\right) \text {, } \\
& \rho=3 \frac{(c-u)^{3 / 2}(c+1+v)^{3 / 2}}{\pi \gamma(1+u+v)^{3}}, \\
& a=\left(\frac{c+1+v}{c-u}\right)^{1 / 2}, \quad b=\left(\frac{c-u}{c+1+v}\right)^{1 / 2} .
\end{aligned}
$$

Region II in which $u \geq 0, v<0$ is the region containing the gravitational waves as described in the previous section. The solution is given by

$$
\begin{aligned}
& U=U_{0}, \quad \sigma=\sigma_{0} \\
& V=V_{1}=c_{n_{1}} \frac{u^{n_{1}}}{\sqrt{v+1}} F\left(\frac{1}{2}, \frac{1}{2}+n_{1} ; 1+n_{1} ;-\frac{u}{v+1}\right), \\
& M=M_{0}+\Omega_{1} .
\end{aligned}
$$

It is understood that terms with different values for $n \geq 1$ can be included in the expression for $V_{1}$, and that $\Omega_{1}$ can be obtained by integrating the equations

$$
\begin{aligned}
& \Omega_{v}=-\frac{1}{2}(1+u+v) V_{v}{ }^{2} \\
& \Omega_{u}=-\frac{1}{2}(1+u+v) V_{u}{ }^{2}
\end{aligned}
$$

using $V=V_{1}$.

The solution in region III $(u<0, v \geq 0)$ can be taken in the same form as in region II, but with $u$ and $v$ interchanged in the expression for $V$, namely,

$$
\begin{aligned}
& U=U_{0}, \quad \sigma=\sigma_{0}, \\
& V=V_{2}=c_{n_{2}} \frac{v^{n_{2}}}{\sqrt{u+1}} F\left(\frac{1}{2}, \frac{1}{2}+n_{2} ; 1+n_{2} ;-\frac{v}{u+1}\right), \\
& M=M_{0}+\Omega_{2} .
\end{aligned}
$$

In the interaction region IV $(u \geq 0, v \geq 0)$, the solution satisfying the necessary junction conditions is given by

$$
\begin{aligned}
& U=U_{0}, \quad \sigma=\sigma_{0}, \\
& V=V_{3}=V_{1}+V_{2}, \\
& M=M_{0}+\Omega_{3},
\end{aligned}
$$


in which the term $V_{3}$ has been taken as the sum of the two expressions given in (6.2) and (6.4) and $\Omega_{3}$ can be obtained from (6.3) using $V_{3}$.

The expansion of the fluid in this region is given by

$$
\begin{aligned}
\theta=\frac{3 \sqrt{2}}{\sqrt{\gamma}} & \frac{[(c-u)(c+v+1)]^{1 / 4}}{(1+u+v)^{3 / 2}} e^{\Omega / 2} \\
\quad & \left((c-u)\left[1+\frac{1}{6}(1+u+v)^{2}{V_{u}}^{2}\right]\right. \\
& \left.\quad+(c+1+v)\left[1+\frac{1}{6}(1+u+v)^{2} V_{v}^{2}\right]\right),
\end{aligned}
$$

where $\Omega=\Omega_{3}$ and $V=V_{3}$. Since $V_{u}$ and $V_{v}$ are bounded in region IV, expression (6.6) indicates that the spacetime after the collision continues to expand indefinitely. Indeed, since $u \in[0, c]$ and $v \in[0, \infty)$ in region IV, Eq. (6.6) shows that $\theta$ remains positive here. Thus, although the presence of the gravitational waves slows down the rate of expansion, future spacelike singulari- ties apparently do not occur for interacting gravitational waves in this expanding background as they do in the vacuum case with a Minkowski background. This result is qualitatively similar to those of Refs. [3,4] which consider the collision of gravitational waves in an expanding vacuum Kasner background. It is also consistent with a theorem of Tipler [19] since, in this case, the singularity is in the past.

\section{ACKNOWLEDGMENTS}

J.B. acknowledges support from Loughborough University of Technology and from the Royal Society which enabled him to visit the Department of Mathematical Sciences in Loughborough, and the Institute of Astronomy in Cambridge for the period in which this work was carried out. J.B. is also grateful to J. B. Griffiths and D. Lynden-Bell for their kind hospitality.
[1] J. Bičák, in Proceedings of the Fifth Marcel Grossmann Meeting on General Relativity, Perth, Australia, 1988, edited by D. G. Blair and M. J. Buckingham (World Scientific, Singapore, 1989), p. 309; see also J. Bičák and B. Schmidt, Phys. Rev. D 40, 1827 (1989).

[2] J. B. Griffiths, Colliding Plane Waves in General Relativity (Oxford University Press, New York, 1991).

[3] J. Centrella, Astrophys. J. 241, 875 (1980).

[4] J. Centrella and R. A. Matzner, Phys. Rev. D 25, 930 (1982).

[5] E. Verdaguer, Phys. Rep. 229, 1 (1993).

[6] P. T. Boyd, J. M. Centrella, and S. A. Klasky, Phys. Rev. D 43, 379 (1991).

[7] M. Carmeli, Ch. Charach, and S. Malin, Phys. Rep. 76, 79 (1981).

[8] P. J. Adams et al., Astrophys. J. 253, 1 (1982).

[9] R. Tabensky and A. H. Taub, Commun. Math. Phys. 29, 61 (1973).

[10] J. Wainwright, Phys. Rev. D 20, 3031 (1979).

[11] J. B. Griffiths, Class. Quantum Grav. 10, 975 (1993).

[12] J. B. Griffiths, J. Math. Phys. 34, 4064 (1993).
[13] C. Misner, K. S. Thorne, and J. A. Wheeler, Gravitation (Freeman, San Francisco, 1973).

[14] J. Wainwright, W. C. W. Ince, and B. J. Marshman, Gen. Relativ. Gravit. 10, 259 (1979).

[15] I. S. Gradshteyn and I. M. Ryzhik, Tables of Integrals, Series and Products (Academic, New York, 1980).

[16] The scalar potential $\sigma$ we are using here was introduced by Wainwright, Ince, and Marshman [14]. It can easily be related (see Ref. [11]) to the potential $\phi$ introduced in the context of a fluid with $p=\rho$ by S. Chandrasekhar and B. C. Xanthopoulos, Proc. R. Soc. London A402, 37 (1985).

[17] S. O'Brien and J. L. Synge, Commun. Dublin Inst. Adv. Stud. A 9 (1952); see also Colliding Plane Waves in General Relativity [2], Chap. 7, for a review of boundary conditions across null hypersurfaces, and references therein.

[18] Handbook of Mathematical Functions, edited by M. Abromowitz and J. A. Stegun (Dover, New York, 1965), p. 560, formula 15.3 .14 .

[19] F. J. Tipler, Phys. Rev. D 22, 2929 (1980). 